\title{
Structural Determinants of Photoreactivity of Triplex Forming Oligonucleotides Conjugated to Psoralens
}

\author{
Rajagopal Krishnan ${ }^{1,2}$ and Dennis H. Oh${ }^{1,2}$ \\ ${ }^{1}$ Department of Dermatology, University of California at San Francisco, San Francisco, CA 94121, USA \\ ${ }^{2}$ Dermatology Research Unit, San Francisco VA Medical Center, 4150 Clement Street, San Francisco, CA 94121, USA
}

Correspondence should be addressed to Dennis H. Oh, ohd@derm.ucsf.edu

Received 15 May 2010; Accepted 3 June 2010

Academic Editor: Ashis Basu

Copyright ( 2010 R. Krishnan and D. H. Oh. This is an open access article distributed under the Creative Commons Attribution License, which permits unrestricted use, distribution, and reproduction in any medium, provided the original work is properly cited.

\begin{abstract}
Triplex-forming oligonucleotides (TFOs) with both DNA and 2'-O-methyl RNA backbones can direct psoralen photoadducts to specific DNA sequences. However, the functional consequences of these differing structures on psoralen photoreactivity are unknown. We designed TFO sequences with DNA and 2'-O-methyl RNA backbones conjugated to psoralen by 2-carbon linkers and examined their ability to bind and target damage to model DNA duplexes corresponding to sequences within the human HPRT gene. While TFO binding affinity was not dramatically affected by the type of backbone, psoralen photoreactivity was completely abrogated by the 2'-O-methyl RNA backbone. Photoreactivity was restored when the psoralen was conjugated to the RNA TFO via a 6-carbon linker. In contrast to the B-form DNA of triplexes formed by DNA TFOs, the CD spectra of triplexes formed with $2^{\prime}$-O-methyl RNA TFOs exhibited features of A-form DNA. These results indicate that $2^{\prime}$-O-methyl RNA TFOs induce a partial B-to-A transition in their target DNA sequences which may impair the photoreactivity of a conjugated psoralen and suggest that optimal design of TFOs to target DNA damage may require a balance between binding ability and drug reactivity.
\end{abstract}

\section{Introduction}

Psoralens plus ultraviolet A (UVA) therapy are widely used in the treatment of psoriasis and other inflammatory skin diseases. Psoralens intercalate at $5^{\prime} \mathrm{TA} 3^{\prime}$ sites and react with thymines upon exposure to UVA. With absorption of the first UVA photon, psoralens form monoadducts (MA), and subsequent exposures may convert furan-sided MA to interstrand crosslinks (XL). This ability to manipulate lesion formation makes psoralens attractive agents for studying DNA damage and repair and for potentially controlling the therapeutic response. Triplex-forming oligonucleotides (TFOs) offer a promising approach to target drugs such as psoralens to specific genes of interest in living cells. However the metabolic activities and electrostatic forces of the cell introduce a major obstacle to this interesting drug delivery system.

In order to overcome barriers to intracellular binding to DNA, TFOs have been extensively engineered. One strategy has been to utilize 2'-O-methyl RNA which has been reported to significantly enhance the binding affinity relative to DNA-based TFOs [1-5]. It has been suggested that the $\mathrm{C}^{\prime}$-endo conformation of the ribose sugar is appropriate for triplex formation $[6,7]$ and that $2^{\prime}$-O-methyl RNA enhances the TFO association rate without hindering the activity of a psoralen conjugated at the $5^{\prime}$ terminus $[1,8]$. However, the $\mathrm{C}^{\prime}$-endo conformation of an RNA TFO also introduces major structural changes in the major groove and in the helical periodicity of the target sequence $[6,9$, 10]. For example, in an intramolecular triplex formed by a $2^{\prime}$-O-methyl RNA pyrimidine-motif TFO, the helical twist increased, and the helical axis was displaced in the purineHoogsteen pair, introducing a dominant A-like structure, when compared with the corresponding DNA TFO, where the dominant structure is B-form [6]. In addition, an RNA third strand has also been reported to induce conformational changes in the sugars of the purine strand of duplex DNA, which resulted in a local B-to A-DNA transition $[11,12]$. Partial B- to A-DNA transitions have also been observed in triplexes formed by $2^{\prime}$-O-methyl RNA strands [13]. These 
TABLE 1: Binding and photoreactive efficiencies for TFOs conjugated to HMT.

\begin{tabular}{lcccc}
\hline TFO & $\begin{array}{c}\text { Nondenaturing } \\
\text { gel }(\mathrm{nM})\end{array}$ & $\begin{array}{c}\text { Denaturing gel } \\
(\mathrm{nM})\end{array}$ & $k_{1}\left(\mathrm{~J} / \mathrm{cm}^{2}\right)^{-1}$ & $19 \pm 0.5$ \\
\hline TFO DNA-C2 & $63 \pm 5$ & $7.08 \pm 2.6$ & 0 & $0.55 \pm 0.04$ \\
TFO RNA-C2 & $75 \pm 18$ & - & $24 \pm 4$ & 0 \\
TFO RNA-C6 & $36 \pm 4$ & $10.96 \pm 2.6$ & $k_{2}\left(\mathrm{~J} / \mathrm{cm}^{2}\right)^{-1}$ & $1.6 \pm 0.05$ \\
\hline
\end{tabular}

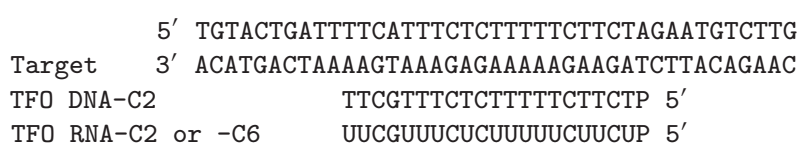

Figure 1: TFO and target DNA sequences. TFOs composed of either DNA or 2'-O-methyl RNA are depicted. P represents HMT conjugated to the TFO by either a 2- or 6-carbon linker.

induced transitions may potentially alter the activity of TFO-conjugated drugs that interact with the DNA. In this work, we studied the effects of the $2^{\prime}$-O-methyl RNA TFO modification on the photoreactivity of psoralens conjugated to them. We observed that relative to the corresponding DNA TFOs, 2'-O-methyl TFOs can dramatically restrict psoralen reactivity in association with a $\mathrm{B}$ - to A-DNA transition in the target sequence.

\section{Materials and Methods}

2.1. Oligonucleotides. Synthetic gel-purified oligonucleotides (Oligos Etc., Wilsonville, OR) used as target sequences are shown in Figure 1. The 41 base pair target sequence corresponds to the sequence of the junction between intron 4 and exon 5 of the human hypoxanthine phosphoribosyltransferase (HPRT) gene, similar to that targeted in Chinese hamster ovary cells [14]. All TFOs were 20 nucleotidelong pyrimidine-motif sequences that bind parallel to the purine strand of the target DNA duplex and that were conjugated through a saturated 2- or 6-carbon linker to the psoralen derivative, $4^{\prime}$-hydroxymethyl trimethylpsoralen (HMT), at their $5^{\prime}$ termini. TFO DNA-C2 contained a DNA backbone with a 2-carbon linker, while RNA-C2 and RNAC6 possessed 2'-O-methyl RNA backbones with 2- and 6carbon linkers, respectively.

2.2. Triplex Formation on Genomic DNA. Human genomic DNA was purified from HT1080 cells by phenol extraction. $10 \mu \mathrm{M}$ TFO DNA-C2 or TFO RNA-C2 were incubated with $1 \mu \mathrm{g}$ genomic DNA in the presence of binding buffer $(10 \mathrm{mM}$ Tris, $\mathrm{pH}$ 7.0, $20 \mathrm{mM} \mathrm{MgCl}_{2}, 1 \mathrm{mM}$ spermidine) at room temperature overnight. The mixtures were then irradiated with $0.2 \mathrm{~J} / \mathrm{cm}^{2}$ UVA and analyzed by single-strand ligation PCR as described previously [15]. Briefly, following UVA treatment, the samples were digested with Dde I which generates a DNA fragment that includes the TFO binding site. Samples were then subject to primer extension with a biotinylated primer ( $5^{\prime}$ Biotin-GGGTTGTTATGATGTGATTTGACTT), and products were captured with streptavidin-coated magnetic beads (Dynal, Oslo, Norway) and ligated at their $3^{\prime}$ termini to a ligation oligonucleotide. The ligation products were then amplified using primers $5^{\prime}$ GTTATGATGTGATTTGACTTATAATTG (primer 2) and 5' TATGACTATGCATGATCTACGAT (ligation primer) and then linearly amplified with ${ }^{32} \mathrm{P}$-end-labeled $5^{\prime}$ GATGTGATTTGACTTATAATTGGAAATA (primer 3 ). The ${ }^{32} \mathrm{P}$ reaction products were separated on a denaturing gel and quantified by phosphorimaging, and relative intensities of the undamaged and adduct site were calculated.

2.3. Target Duplex DNA and Triplex Formation. Synthetic duplex DNA targets containing the TFO binding sites were formed by annealing the complementary target sequences shown in Figure 1. Prior to annealing, the purine strand of the target duplex was labeled at the $5^{\prime}$-terminus with ${ }^{32} \mathrm{P}$ using T4 polynucleotide kinase (GE Healthcare, Piscataway, NJ). Equimolar quantities of complementary oligonucleotides were mixed in $10 \mathrm{mM}$ Tris, $\mathrm{pH} 7.5,1 \mathrm{mM}$ EDTA, heated to $95^{\circ} \mathrm{C}$ for $5 \mathrm{~min}$, and cooled to room temperature over several hours. $5 \mathrm{nM}$ of the resulting duplex was incubated with TFOs in binding buffer at room temperature overnight. The equilibrium products (duplex and triplex) were separated on a $6 \%$ neutral nondenaturing polyacrylamide gel and quantified by phosphorimaging (Bio-Rad GS-360). The dissociation constant, $K_{d}$, was obtained by fitting the bound fraction to a two-state model, as previously described [16]. For studies of photoreactivity, the equilibrium TFO-duplex binding mixture was irradiated with UVA as described previously [16]. The products were separated on a $6 \%$ denaturing polyacrylamide gel, quantified by phosphor imaging and an apparent $K_{d}$ and the photoefficiency constants for MA and XL formation, $k_{1}$ and $k_{2}$, respectively, were obtained by analyzing the product fraction as described before [16].

2.4. Circular Dichroism (CD). DNA duplex and triplex samples without ${ }^{32} \mathrm{P}$ labeling were prepared as described above and transferred to a $2 \mathrm{~mm}$ pathlength cuvette. CD spectra were measured in a JASCO J-710 spectropolarimeter equipped with a Xenon lamp. The data from $210 \mathrm{~nm}$ to $320 \mathrm{~nm}$ were recorded with a sensitivity of $100 \mathrm{mdeg}, 1 \mathrm{~nm}$ bandwidth, $0.1 \mathrm{~nm}$ stepsize, and scan speed of $20 \mathrm{~nm} / \mathrm{min}$. The spectral data reported are the average of 5 scans. 

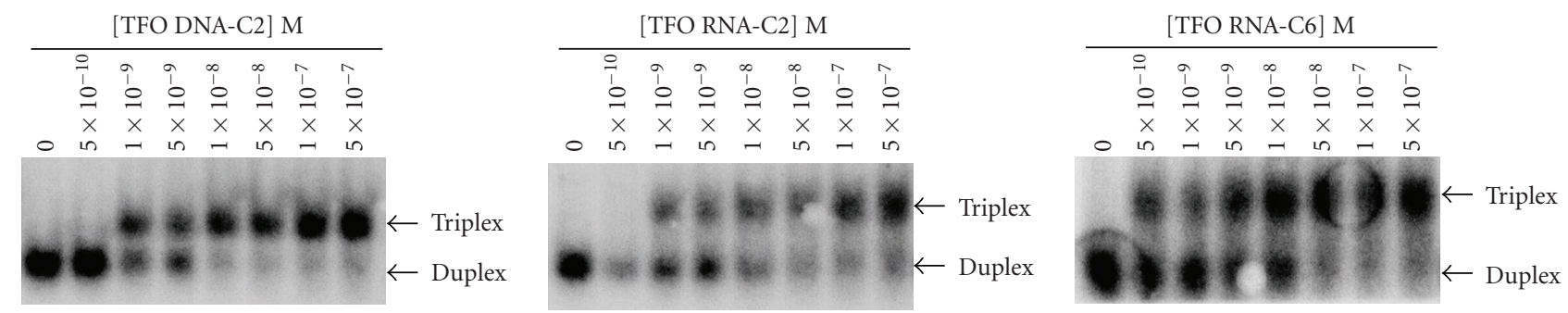

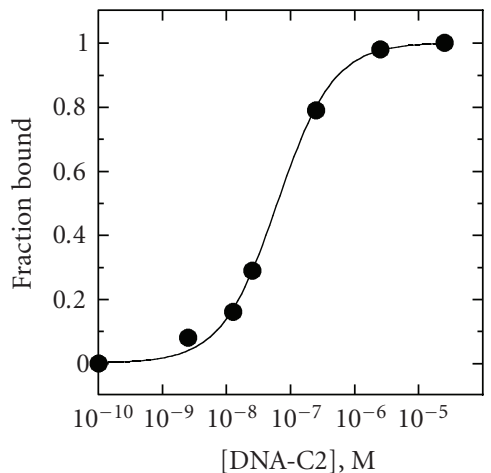

(a)

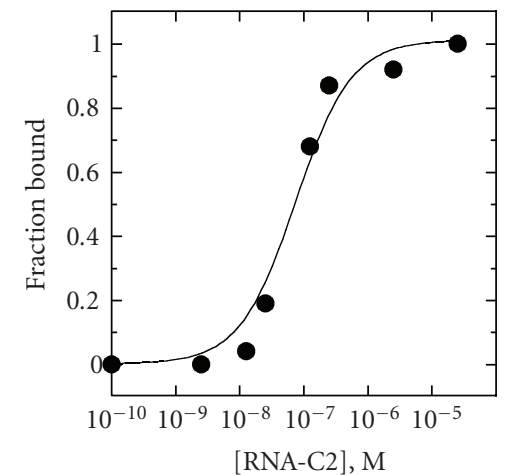

(b)

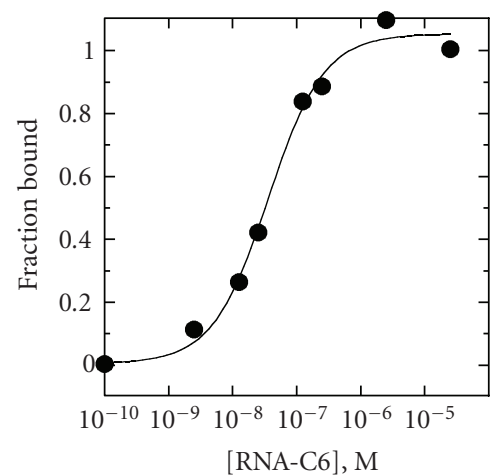

(c)

FIGURE 2: Noncovalent binding of TFOs to duplex target. Varying concentrations of (a) TFO DNA-C2, (b) TFO RNA-C2, and (c) TFO RNAC6 were incubated with $5 \mathrm{nM}$ target DNA duplex overnight before polyacrylamide gel electrophoresis under nondenaturing conditions. Gel bands were quantified and the data was fit with a two-state binding model.

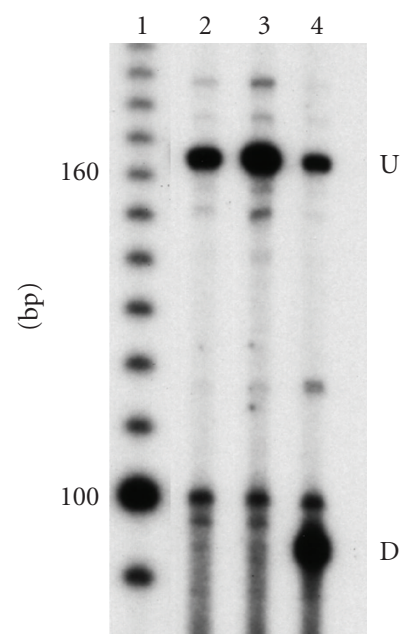

Figure 3: Binding of TFOs to genomic DNA targets. $2 \mu \mathrm{M}$ of TFO, either TFO RNA-C2 (lanes 2 and 3) or TFO DNA-C2 (lane 4) were incubated with genomic DNA purified from HT1080 cells and mock irradiated (lane 2) or irradiated with $0.2 \mathrm{~J} / \mathrm{cm}^{2}$ UVA (lanes 3 and 4) and analyzed by single-strand ligation PCR to assay for undamaged (U) and site-specifically damaged (D) DNA. Lane 1 is a 10 base pair DNA ladder.

\section{Results and Discussion}

3.1. 2'-O-Methyl RNA Backbones Minimally Affects TFO Binding. As shown in Figure 1, TFOs were designed to target the junction between intron 4 and exon 5 of the human
HPRT gene. TFO DNA-C2 is a DNA TFO conjugated to HMT at the $5^{\prime}$ end with 2-carbon linker. TFO RNA-C2 is the corresponding $2^{\prime}$-O-methyl RNA TFO. TFO DNA-C2, when equilibrated with the synthetic DNA duplex target, caused a near-quantitative mobility shift on nondenaturing gel electrophoresis, indicating triplex formation (Figure 2). Quantification of band intensities indicated that TFO DNAC2 bound with reasonable affinity to the DNA target (Figure 2, Table 1). Under the experimental conditions used, the observed $K_{d}$ value of $63 \mathrm{nM}$ for binding of TFO DNA-C2 to human HPRT target is comparable to previously observed values of about $130 \mathrm{nM}$ in rodent DNA $[8,17]$.

A number of prior reports, however, have indicated that the $3^{\prime}$-endo form of the ribose sugar in an RNA TFO enhances the TFOs binding affinity relative to the corresponding deoxyribose backbone [1-8]. This binding enhancement was attributed to the $3^{\prime}$-endo form being the optimum conformation for Hoogsteen base pairing [6]. Relative to the DNA TFO, the RNA TFO introduces other structural modifications that further enhance TFO binding ability $[1-3,5,8]$. For example, the $2^{\prime}$-O-methyl group positioned between the TFO and duplex purine strand favors the formation of additional van der Waal contacts $[6,18]$.

In an attempt to improve TFO binding, TFOs RNA-C2 and RNA-C6 were designed to possess $2^{\prime}$-O-methyl ribose backbones and uracil bases instead of thymine and were conjugated to HMT via 2-or 6-carbon linkers, respectively. Although the $2^{\prime}$-O-methyl modified TFOs were expected to bind with higher affinity to the duplex DNA target $[1,5,8]$, they actually exhibited binding affinities quite similar to that 


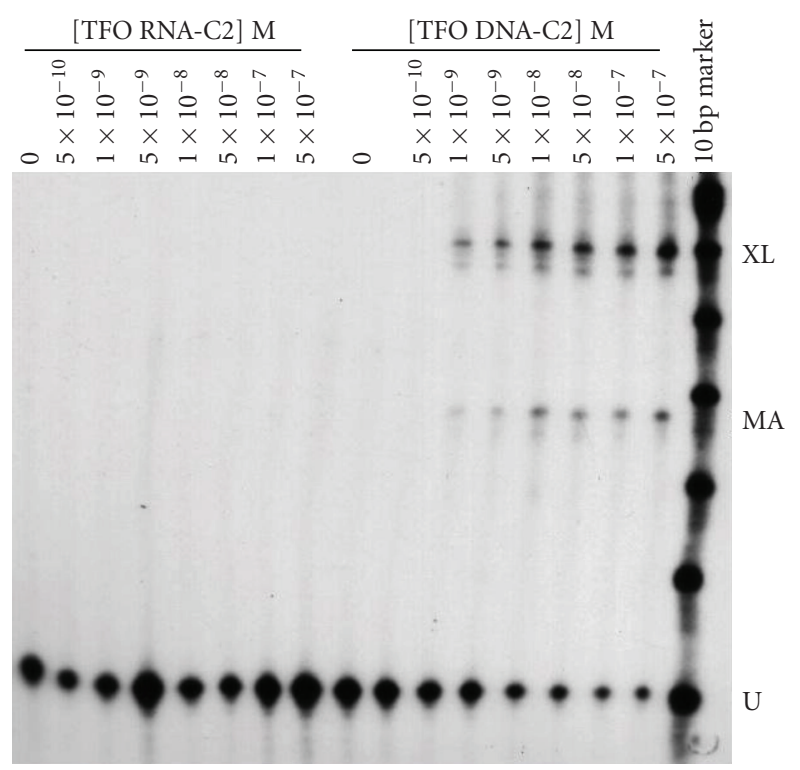

(a)

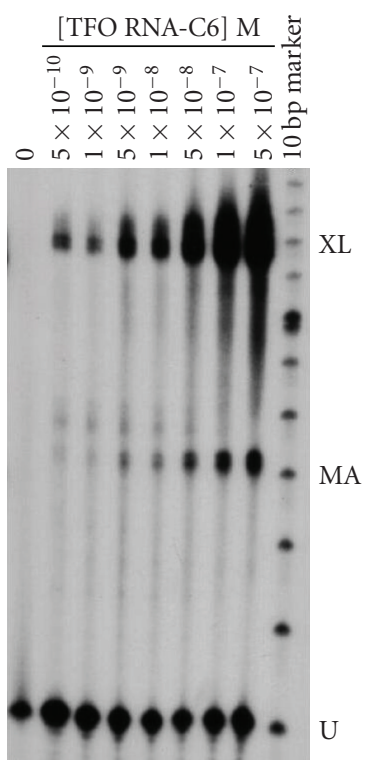

(b)

FIGURE 4: Covalent binding of TFOs to duplex target following UVA. Varying concentrations of (a) TFO DNA-C2 or TFO RNA-C2 or of (b) TFO RNA-C6 were incubated with $5 \mathrm{nM}$ target overnight prior to irradiation with $2 \mathrm{~J} / \mathrm{cm}^{2} \mathrm{UVA}$. The products were then analyzed by polyacrylamide gel electrophoresis under denaturing conditions and denoted as unreacted (U), monoadducts (MA), and crosslinks (XL).

of TFO DNA-C2 (Figure 2, Table 1). While TFO RNA-C2 had a $K_{d}$ that was not significantly different from that of TFO DNA-C2, a modest reduction in $K_{d}$ was observed for TFO RNA-C6, suggesting that the 6-carbon linker further stabilizes binding by allowing better psoralen intercalation, or less likely through direct interactions with the triplex structure, consistent with observations in DNA TFOs [19]. This lack of dramatic enhancement in binding affinity of RNA third strands binding to DNA duplexes has also been observed previously by Han and Dervan [20] and may be due to the RNA TFO's lack of the C-5 methyl group in uridine which likely enhances base stacking and triplex stability [1$3,18,21,22]$.

\subsection{2'-O-Methyl RNA Backbones Affect HMT-TFO Photore-} activity. To detect the binding of TFOs to genomic DNA, we incubated TFOs with human genomic DNA, irradiated the binding mixtures with UVA, and assayed for site-specific psoralen photoadducts using single-stranded ligation PCR. The particular conditions and primers used would be expected to produce PCR amplification products of 163 base pairs for undamaged DNA and of 95 base pairs for DNA damaged by psoralen photoadducts at the TFO target site.

As shown in Figure 3, in the absence of UVA, no site-specific adducts were detected. Following UVA irradiation, TFO DNA-C2 treatment resulted in site-specific photoadduct formation in a significant fraction (87\%) of the genomic DNA. In contrast, TFO RNA-C2 treatment followed by UVA irradiation resulted in a barely detectable band at the expected photoadduct site that constituted a much smaller fraction of the total genomic DNA, indicating that little DNA damage was targeted by TFO RNA-C2. Since the singlestrand ligation PCR assay does not clearly discriminate between MA and XL formation and poor binding, we examined the photoproducts more carefully.

Binding of TFOs conjugated to HMT allows the HMT to react with adjacent target thymine. The HPRT duplex target sequence possesses a $5^{\prime} \mathrm{TpA} 3^{\prime}$ site immediately adjacent to the $5^{\prime}$ terminus of the TFOs, potentially allowing both MA and XL photoadducts to form upon TFO binding and UVA exposure. UVA exposure thus allows covalent capture of all triplex structures in which psoralen photochemistry is possible. Following binding of TFOs to the duplex target, samples were irradiated with UVA and the products were analyzed by denaturing gel electrophoresis. As shown in Figure 4(a), TFO DNA-C2 created dose-dependent covalent photoadducts corresponding to MA and XL. The $K_{d}$ for TFO DNA-C2 that was obtained from analysis of photoproducts in denaturing electrophoretic gels was almost ten-fold lower than that obtained from non-denaturing gels (Table 1). The difference in $K_{d}$ may signify that psoralen photoadduct formation drives additional binding.

In contrast, upon UVA irradiation, TFO RNA-C2 exhibited a complete inability to deliver photoadducts to the target sequence (Figure $4(\mathrm{a})$ ). TFO concentrations up to $10 \mu \mathrm{M}$ were ineffective in producing detectable MA or XL (data not shown). However, photoreactivity could be restored by increasing the linker length between TFO and HMT moieties (Figure 4(b)). TFO RNA-C6 generated dose-dependent MA and XL comparable to TFO DNA-C2, and analysis of the covalent photoadducts resulted in an apparent $K_{d}$ that was also similar to TFO DNA-C2. The dependence of photoreactivity when going from 2- to 6-carbon linkers in RNA 


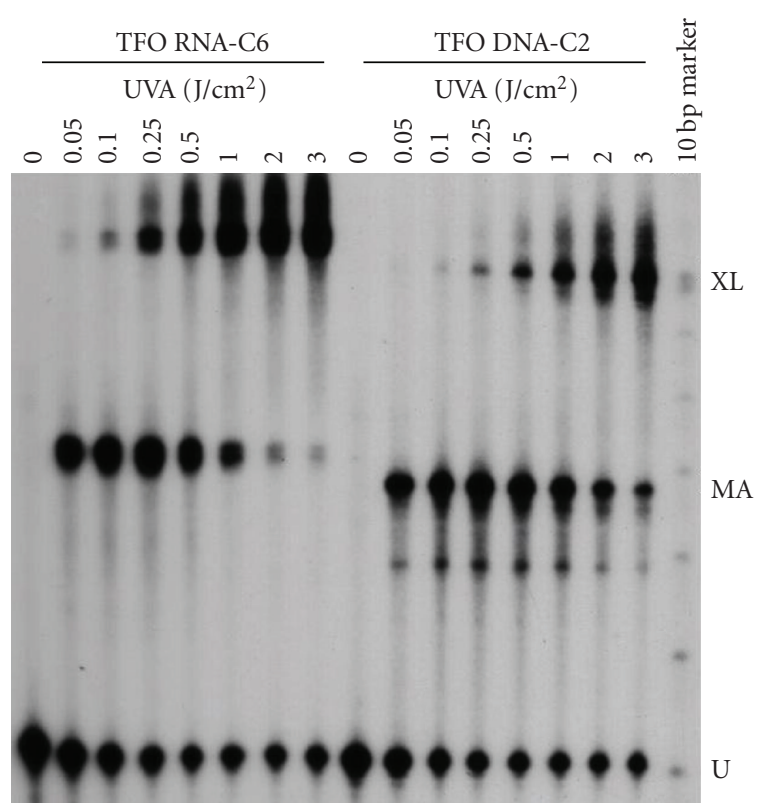

(a)

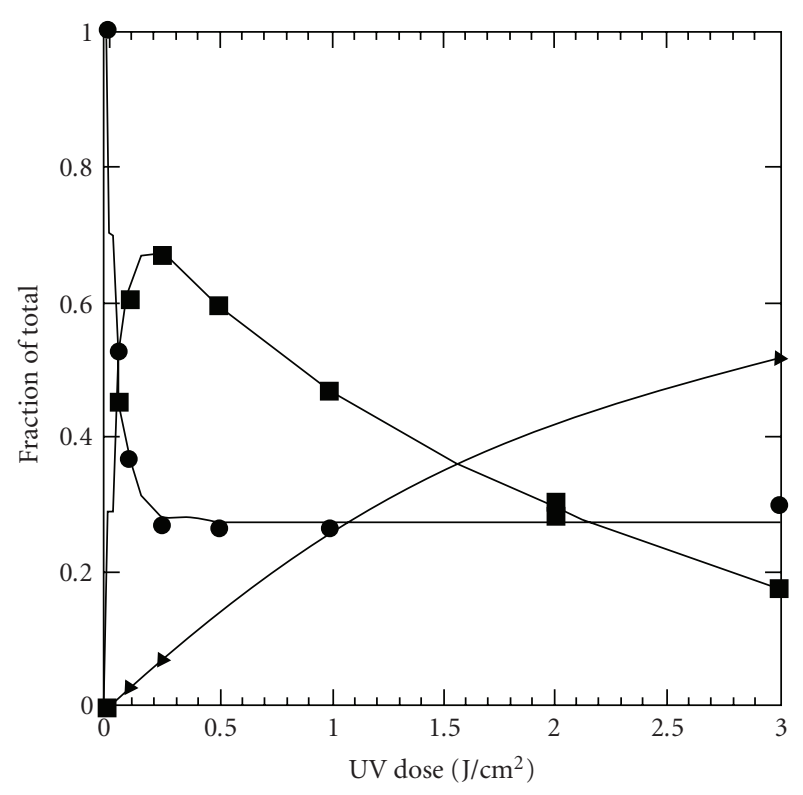

(b)

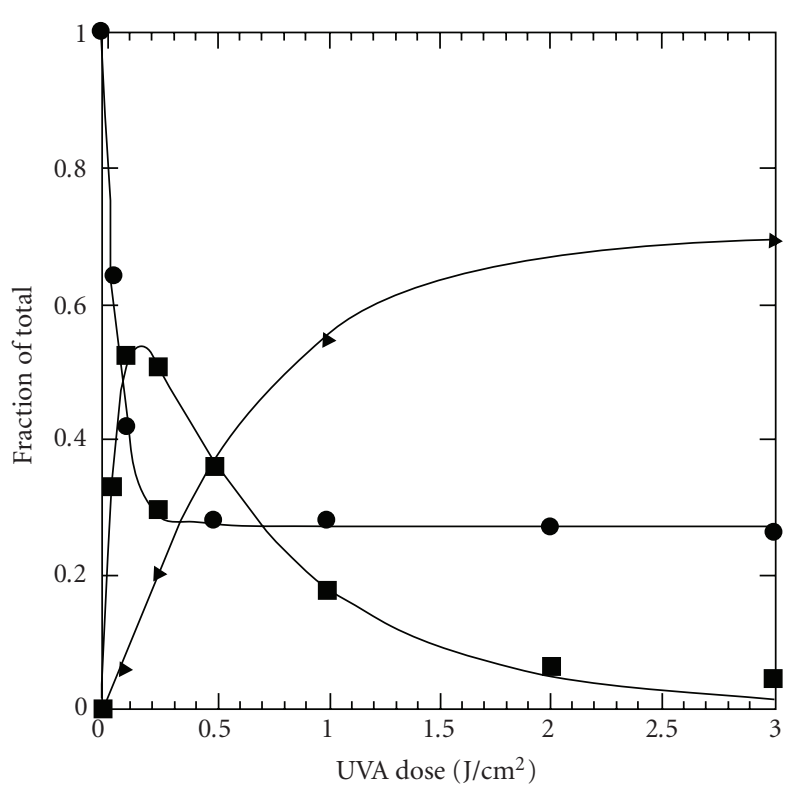

(c)

FIgURE 5: Photoreactivity of TFOs conjugated to HMT. $50 \mathrm{nM}$ TFO DNA-C2 or TFO RNA-C6 were incubated with $5 \mathrm{nM}$ duplex target overnight and then irradiated with varying doses of UVA. (a) Separation of reaction products by polyacrylamide gel electrophoresis under denaturing conditions. The products are marked as unreacted (U), monoadducts (MA), and crosslinks (XL). Quantitative analysis (b) TFO DNA-C2 and (c) TFO RNA-C6 for unreacted DNA (circles), MA (squares), and XL (triangles).

TFOs is consistent with a previously reported dependence of mutagenesis on linker length in DNA TFOs [19]. Our results indicate that although TFO backbone composition does not markedly alter TFO binding affinity to the target, it can profoundly affect psoralen photoreactivity.

To assess TFO photoreactivity in further detail, we exposed TFOs bound to the duplex DNA target to varying UVA doses and analyzed the resulting MA and XL photoadducts as a function of UVA dose by modeling the data with a standard model for psoralen photokinetics [16, 23]. Both TFO DNA-C2 and TFO RNA-C6 reacted with the target DNA duplex by first forming MAs that converted to XLs with subsequent UVA excitation (Figure 5(a)). While MAs formed at approximately similar efficiencies $\left(k_{1}\right)$, XL formation $\left(k_{2}\right)$ was almost 3-fold more efficient with TFO RNA-C6 (Figures 5(b) and 5(c), Table 1). The ability of a 6-carbon linker to restore psoralen photoreactivity indicates that the $2^{\prime}$-O-methyl sugar is likely not simply causing the 


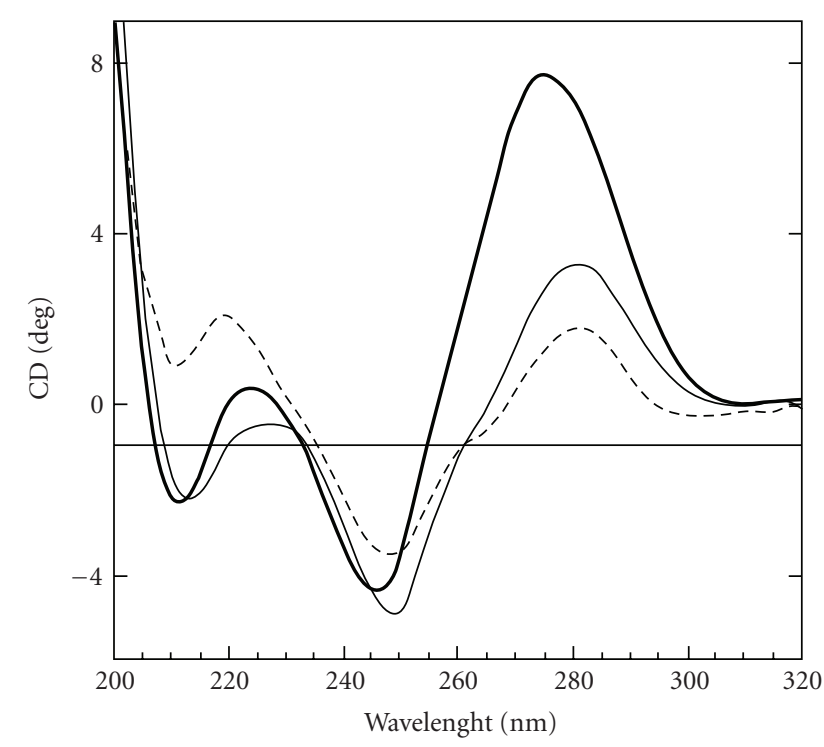

Figure 6: CD spectra of TFOs bound to duplex DNA. Shown are spectra for target DNA duplex alone (dashed line) or duplex incubated with TFO DNA-C2 (thin line) or TFO RNA-C2 (bold line).

TpA site to become locally sterically inaccessible. Rather, we speculated that a more global change occurs upon $2^{\prime}$ $O$-methyl RNA TFO binding that renders the TpA site inaccessible to psoralen when tethered to TFO via a 2 -carbon linker, but not a 6-carbon linker.

3.3. Distortion from B-Form DNA upon Binding of RNA TFOs. We hypothesized that our 2'-O-methyl RNA TFOs induce a local A-DNA structure in the duplex DNA target under our triplex-forming conditions, as has been previously described for RNA TFOs bound to DNA duplexes [11, 12]. To specifically identify structural changes to the triple helix that might hinder psoralen intercalation and/or photoreactivity, we measured circular dichroism (CD) spectra of our triplexes. As expected and observed previously by others the free DNA and RNA TFO showed CD spectra corresponding to B- and A-Form, respectively [24-26]. The CD spectrum of both the target DNA duplex alone and triplexes formed by target duplex and TFO DNA-C2 each exhibited both a positive peak at $280 \mathrm{~nm}$ and a negative peak near $250 \mathrm{~nm}$, and these peak intensities were conserved due to the Cotton effect, indicating that both the DNA duplex and triplex are predominantly in the B-form (Figure 6) [26-29]. If present, the local B- to A-DNA transition might require a large reorientation of psoralen to intercalate into the target site, hindering the psoralen activity. In contrast, the CD spectrum of triplexes formed by TFO RNA-C2 exhibited shifts in the positive peak to $270 \mathrm{~nm}$ while the negative peak shifted to $245 \mathrm{~nm}$, and the peaks were non-conserved, indicative of base tilting or distortion. These spectral features are characteristic of A-DNA in which the bases are oriented in a twisted propeller rather than planar configuration [18, 2729]. In addition, the results are consistent with prior CD data indicating the presence of the A-DNA conformation in an intramolecular triplex formed with DNA duplex part and $2^{\prime}$-O-methyl RNA third strand [13]. While NMR studies conducted on a similar triplex did not observe A-DNA in $2^{\prime}$ O-methyl RNA-DNA triplexes [6], the number of base pairs per helical twist was found to be near $11 \mathrm{bp}$ which is close to that of A-DNA [6].

In any case, our results indicate that the $2^{\prime}$-O-methyl RNA TFO induces helical transitions that are associated with reduced photoreactivity of psoralen when it is conjugated to the TFO with a 2-carbon linker. Because the helical periodicity increases from 10.5 base pairs per helical turn in B-DNA to 11 base pairs per helical turn in A-DNA, we speculate that the A-form DNA conformations induced by the RNA TFOs move the TpA site out of range of the psoralen when it is conjugated to the TFO by a 2-carbon linker, but not a 6-carbon linker.

\section{Conclusions}

TFOs are promising agents for targeting DNA damage to specific sites in the genome and may be exploited to introduce sequence specific mutations or to alter gene expression [29-31]. The conjugation of psoralens to TFOs can enhance these effects [29]. In general, the enhancement of binding affinity as well as the stability of TFOs by modifications of the sugar and base structure is anticipated to translate into better targeting of DNA damage and biological effects. However, in some cases, enhanced binding affinity in vitro due to chemical modifications to the backbone and bases has been associated with diminished biological activity from the conjugated psoralen [7]. Our results indicate that at least certain TFO modifications can result in structural changes that impair psoralen photoreactivity. Therefore, in certain cases, optimal design of TFOs to target DNA damage by psoralen may require a balance between maximizing binding ability and preserving drug reactivity. An additional consideration is that the structural changes induced by different TFO structures may also be differentially recognized and processed by DNA repair pathways.

\section{Acknowledgments}

This work was supported by NIH Grant RO1CA105958 to D. H. Oh. The authors thank Dr. David Agard and Neema Salimi use of the CD spectrometer and assistance with CD measurements. They also thank Christina Butcher for technical assistance and the rest of the Oh Laboratory for helpful discussions.

\section{References}

[1] S. Wang and E. T. Kool, "Origins of the large differences in stability of DNA and RNA helices: C-5 methyl and 2'-hydroxyl effects," Biochemistry, vol. 34, no. 12, pp. 4125-4132, 1995.

[2] M. Shimizu, T. Koizumi, H. Inoue, and E. Ohtsuka, "Effects of 5-methyl substitution in 2'-O-methyloligo(pyrimidine) nucleotides on triple-helix formation," Bioorganic and Medicinal Chemistry Letters, vol. 4, no. 8, pp. 1029-1032, 1994. 
[3] C. Escudeé, J.-C. Francçois, J. S. Sun et al., "Stability of triple helices containing RNA and DNA strands: experimental and molecular modeling studies," Nucleic Acids Research, vol. 21, no. 24, pp. 5547-5553, 1993.

[4] R. W. Roberts and D. M. Crothers, "Stability and properties of double and triple helices: dramatic effects of RNA or DNA backbone composition," Science, vol. 258, no. 5087, pp. 1463 1466, 1992.

[5] M. Shimizu, A. Konishi, Y. Shimada, H. Inoue, and E. Ohtsuka, "Oligo(2'-O-methyl)ribonucleotides: effective probes for duplex DNA," FEBS Letters, vol. 302, no. 2, pp. 155-158, 1992.

[6] J. L. Asensio, R. Carr, T. Brown, and A. N. Lane, "Conformational and thermodynamic properties of parallel intramolecular triple helices containing a DNA, RNA, or 2'-OMeDNA third strand," Journal of the American Chemical Society, vol. 121, no. 48, pp. 11063-11070, 1999.

[7] M. D. R. Alam, A. Majumdar, A. K. Thazhathveetil et al., "Extensive sugar modification improves triple helix forming oligonucleotide activity in vitro but reduces activity in vivo," Biochemistry, vol. 46, no. 35, pp. 10222-10233, 2007.

[8] N. Puri, A. Majumdar, B. Cuenoud et al., "Minimum number of 2r-O-(2-aminoethyl) residues required for gene knockout activity by triple helix forming oligonucleotides," Biochemistry, vol. 41, no. 24, pp. 7716-7724, 2002.

[9] J. L. Asensio, T. Brown, and A. N. Lane, "Solution conformation of a parallel DNA triple helix with 5' and 3' triplex-duplex junctions," Structure, vol. 7, no. 1, pp. 1-11, 1999.

[10] C. H. Gotfredsen, P. Schultze, and J. Feigon, "Solution structure of an intramolecular: pyrimidine-purine-pyrimidine triplex containing an RNA third strand," Journal of the American Chemical Society, vol. 120, no. 18, pp. 4281-4289, 1998.

[11] C. Dagneaux, J. Liquier, and E. Taillandier, "Sugar conformations in DNA and RNA-DNA triple helices determined by FTIR spectroscopy: role of backbone composition," Biochemistry, vol. 34, no. 51, pp. 16618-16623, 1995.

[12] J. Liquier, E. Taillandier, R. Klinck, E. Guittet, C. Gouyette, and T. Huynh-Dinh, "Spectroscopic studies of chimeric DNARNA and RNA 29-base intramolecular triple helices," Nucleic Acids Research, vol. 23, no. 10, pp. 1722-1728, 1995.

[13] E. R. Kandimalla, G. Venkataraman, V. Sasisekharan, and S. Agrawal, "Single-stranded DNA and RNA targeted triplexformation: UV, CD and molecular modeling studies of foldback triplexes containing different RNA, 2'-OMe-RNA and DNA strand combinations," Journal of Biomolecular Structure and Dynamics, vol. 14, no. 6, pp. 715-726, 1997.

[14] A. Majumdar, A. Khorlin, N. Dyatkina et al., "Targeted gene knockout mediated by triple helix forming oligonucleotides," Nature Genetics, vol. 20, no. 2, pp. 212-214, 1998.

[15] D. H. Oh and P. C. Hanawalt, "Triple helix-forming oligonucleotides target psoralen adducts to specific chromosomal sequences in human cells," Nucleic Acids Research, vol. 27, no. 24, pp. 4734-4742, 1999.

[16] D. H. Oh and P. C. Hanawalt, "Binding and photoreactivity of psoralen linked to triple helix-forming oligonucleotides," Photochemistry and Photobiology, vol. 72, no. 3, pp. 298-307, 2000.

[17] N. Puri, A. Majumdar, B. Cuenoud et al., "Targeted gene knockout by 2'-O-aminoethyl modified triplex forming oligonucleotides," The Journal of Biological Chemistry, vol. 276, no. 31, pp. 28991-28998, 2001.
[18] J.-S. Sun and C. Hélène, "Oligonucleotide-directed triple-helix formation," Current Opinion in Structural Biology, vol. 3, no. 3, pp. 345-356, 1993.

[19] M. Raha, L. Lacroix, and P. M. Glazer, "Mutagenesis mediated by triple helix-forming oligonucleotides conjugated to psoralen: effects of linker arm length and sequence context," Photochemistry and Photobiology, vol. 67, no. 3, pp. 289-294, 1998.

[20] H. Han and P. B. Dervan, "Sequence-specific recognition of double helical RNA and RNA. DNA by triple helix formation," Proceedings of the National Academy of Sciences of the United States of America, vol. 90, no. 9, pp. 3806-3810, 1993.

[21] S. Wang, Y. Xu, and E. T. Kool, "Recognition of RNA by tripler formation: divergent effects of pyrimidine C-5 methylation," Bioorganic and Medicinal Chemistry, vol. 5, no. 6, pp. 10431050, 1997.

[22] R. A. Cassidy, N. Puri, and P. S. Miller, "Effect of DNA target sequence on triplex formation by oligo-2'-deoxy- and 2'-Omethylribonucleotides," Nucleic Acids Research, vol. 31, no. 14, pp. 4099-4108, 2003.

[23] G. D. Cimino, H. B. Gamper, S. T. Isaacs, and J. E. Hearst, "Psoralens as photoactive probes of nucleic acid structure and function: organic chemistry, photochemistry, and biochemistry," Annual Review of Biochemistry, vol. 54, no. 1, pp. 11511193, 1985.

[24] S.-H. Hung, Q. Yu, D. M. Gray, and R. L. Ratliff, "Evidence from $\mathrm{CD}$ spectra that $\mathrm{d}$ (purine) $\mathrm{r}$ (pyrimidine) and $\mathrm{r}$ (purine) $\cdot \mathrm{d}$ (pyrimidine) hybrids are in different structural classes," Nucleic Acids Research, vol. 22, no. 20, pp. 4326-4334, 1994.

[25] C. L. Clark, P. K. Cecil, D. Singh, and D. M. Gray, “CD, absorption and thermodynamic analysis of repeating dinucleotide DNA, RNA and hybrid duplexes $[\mathrm{d} / \mathrm{r}(\mathrm{AC})]_{12} \cdot[\mathrm{d} / \mathrm{r}(\mathrm{GT} / \mathrm{U})]_{12}$ and the influence of phosphorothioate substitution," Nucleic Acids Research, vol. 25, no. 20, pp. 4098-4105, 1997.

[26] J. T. Yang and T. Samejima, "Effect of base tilting on the optical activity of nucleic acids: a hypothesis," Biochemical and Biophysical Research Communications, vol. 33, no. 5, pp. 739745, 1968.

[27] V. I. Ivanov, L. E. Minchenkova, A. K. Schyolkina, and A. I. Poletayev, "Different conformations of double-stranded nucleic acid in solution as revealed by circular dichroism," Biopolymers, vol. 12, no. 1, pp. 89-110, 1973.

[28] W. C. Johnson and I. Tinoco, "Circular dichroism of polynucleotides: a simple theory,” Biopolymers, vol. 7, no. 5, pp. 727$749,1969$.

[29] K. M. Knee, S. B. Dixit, C. E. Aitken, S. Ponomarev, D. L. Beveridge, and I. Mukerji, "Spectroscopic and molecular dynamics evidence for a sequential mechanism for the A-toB transition in DNA," Biophysical Journal, vol. 95, no. 1, pp. 257-272, 2008.

[30] S. A. Strobel, L. A. D. Stamm, L. Riba, D. E. Housman, and P. B. Dervan, "Site-specific cleavage of human chromosome 4 mediated by triple-helix formation," Science, vol. 254, no. 5038, pp. 1639-1642, 1991.

[31] C. Giovannangeli, S. Diviacco, V. Labrousse, S. Gryaznov, P. Charneau, and C. Helene, "Accessibility of nuclear DNA to triplex-forming oligonucleotides: the integrated HIV-1 provirus as a target," Proceedings of the National Academy of Sciences of the United States of America, vol. 94, no. 1, pp. 7984, 1997. 

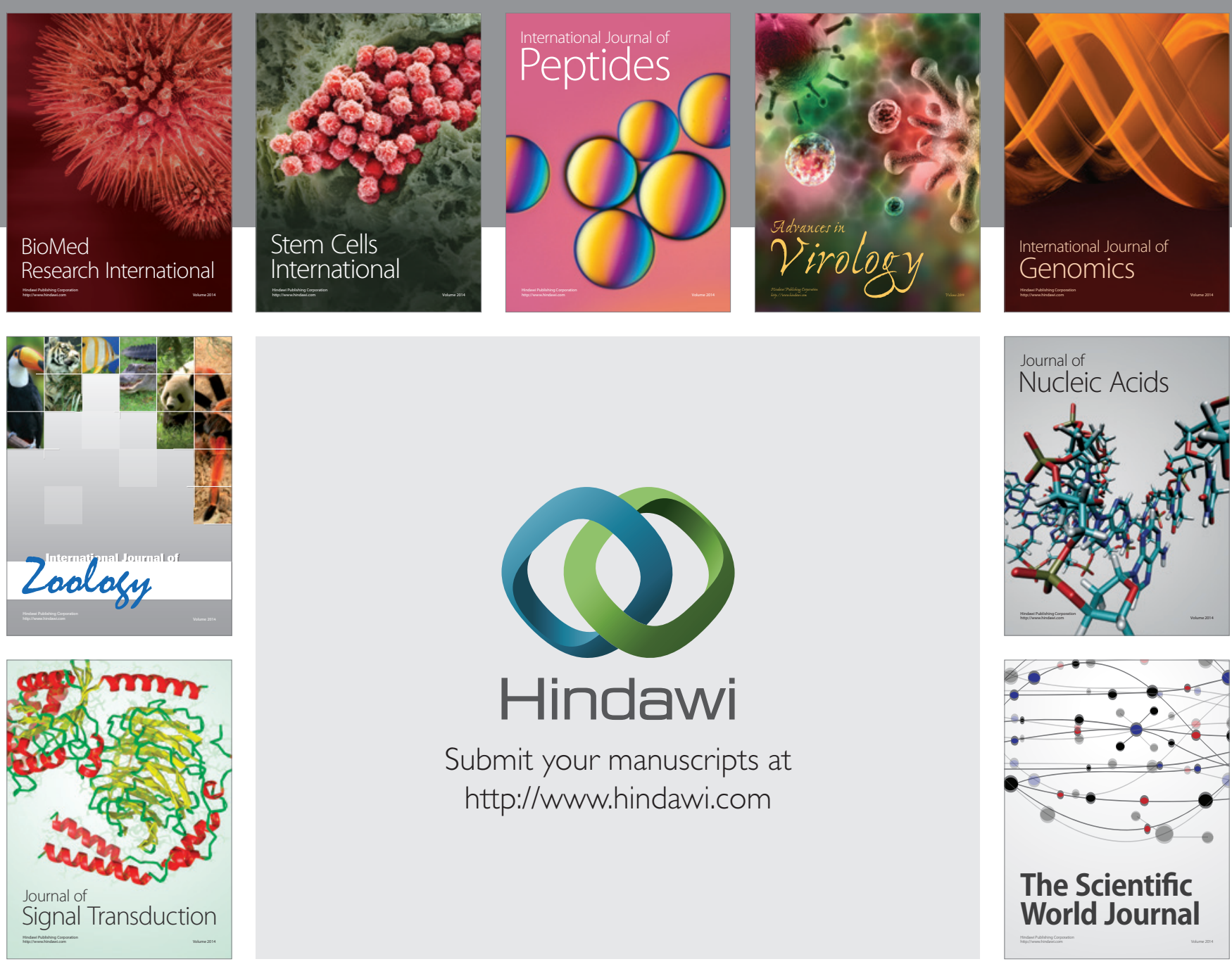

Submit your manuscripts at

http://www.hindawi.com
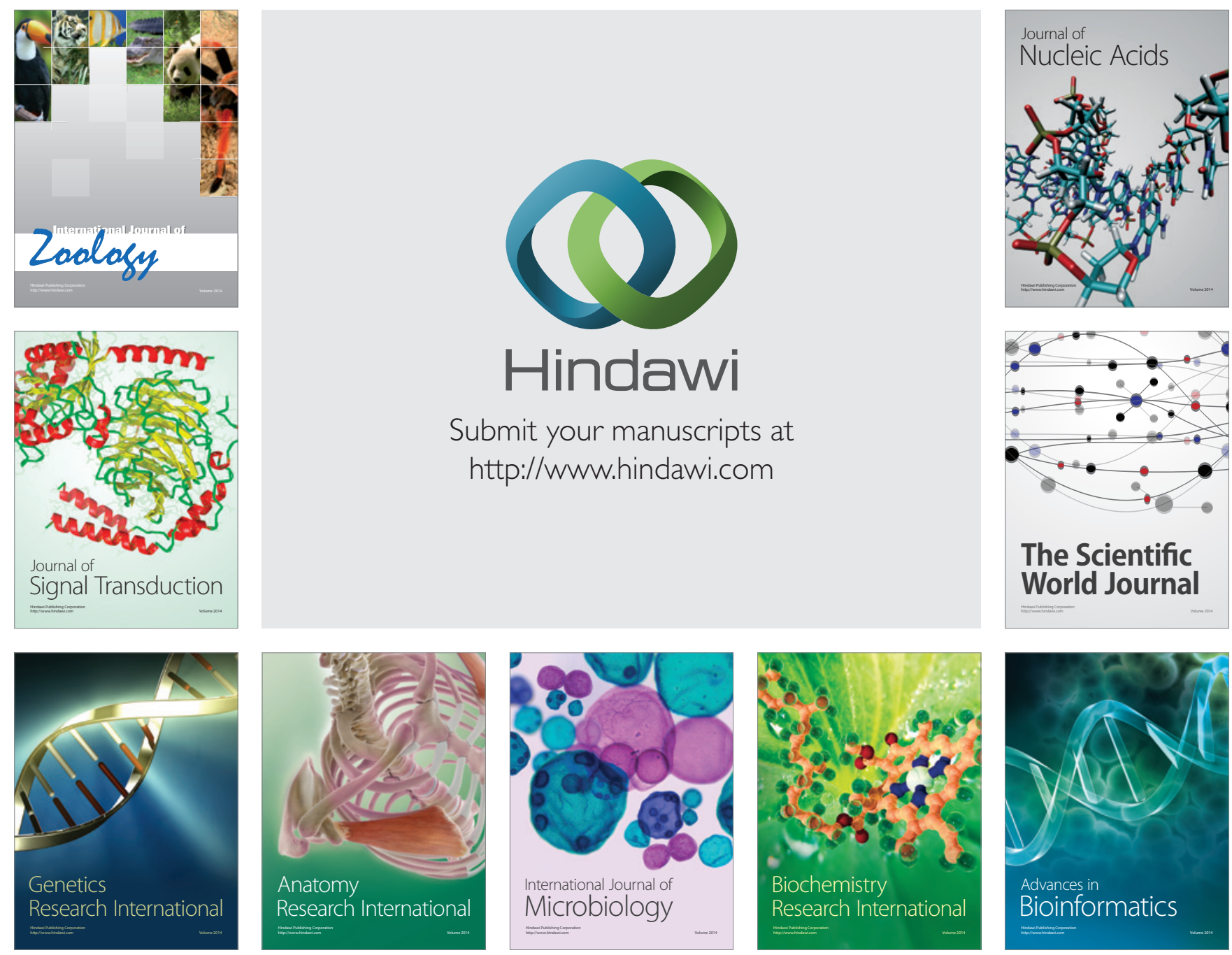

The Scientific World Journal
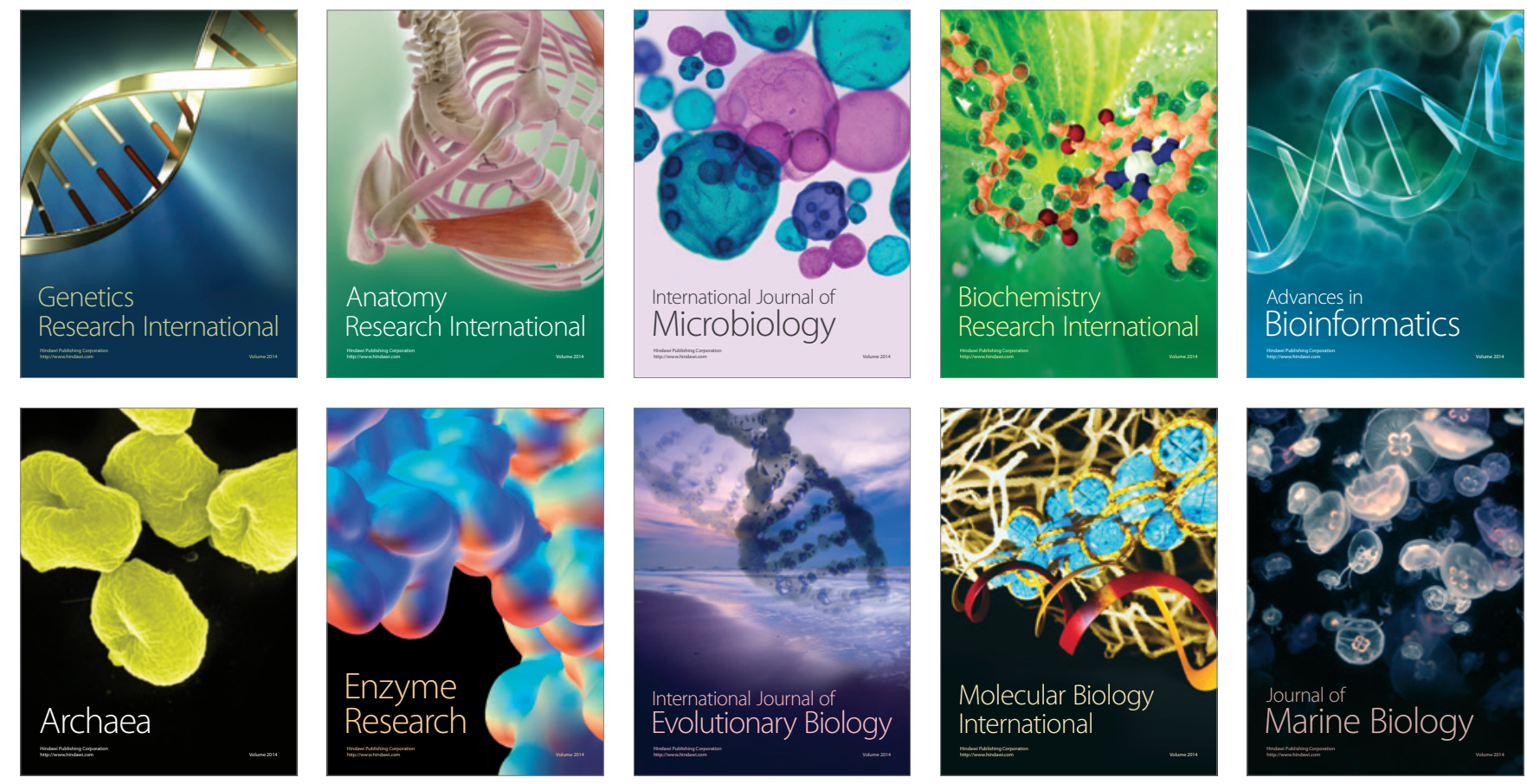